\title{
Well Defined Rich Electronic Structure: Facile Approach for Nido-Carborane Fused Azaspirodecaniums in Water Solution
}

\author{
Zhixiang Lv, ${ }^{a}$ Zhou Wang, ${ }^{b}$ Fuyan Xiao ${ }^{c}$ and Guofan Jin ${ }^{\circledR} * c$ \\ ${ }^{a}$ Department of Pharmacy, Danyang People's Hospital, 212300 Danyang, P. R. China \\ ${ }^{b}$ College of Vanadium and Titanium, Panzhihua University, 617000 Panzhihua, P. R. China \\ 'School of Pharmacy, Jiangsu University, 212013 Zhenjiang, P. R. China
}

\begin{abstract}
A novel bis-dumbbell shape of a multi cation around a azaspirodecanium combined with nido-carborane was prepared in a single step in good yield starting from pyrrolidine and 1,3-dichloropropane to give one compound and from piperidine and 1,4-dichlorobutane to give the other. This is the first report of an electrolyte azaspirodecanium-boron-cluster structure. ${ }^{1} \mathrm{H},{ }^{13} \mathrm{C}$, and ${ }^{11} \mathrm{~B}$ nuclear magnetic resonance spectroscopy, Fourier transform infrared spectroscopy, and high resolution mass spectrometry (HRMS) methods revealed changes due to interactions among the functional groups. In particular, the compound was electron-rich because of its unique structure and excellent chemical reactivity.
\end{abstract}

Keywords: nido-carborane, azaspirodecaniums, boron-cluster structure

\section{Introduction}

Nido-carborane ${ }^{1}$ is a type of loose one boron atom defect with an open type basket structure; its precursor carborane structure is composed of ten embedded boron atoms with empty electron orbitals and two carbon atoms, confirming its active reaction. ${ }^{2,3}$ This structure plays an important role in the field of organic and inorganic chemistry, and is widely used in scientific research, such as metal and non-metal chelation. ${ }^{4}$ For example, in organic medicine, the boron neutron-capture therapy (BNCT) treatment method uses an externally irradiated thermal neutron source to excite boron atoms to generate energy levels to destroy tumor cells and achieve the therapeutic goals. ${ }^{5-11}$ In the inorganic and organic metallic directions, the physical properties of electron transfer and orbital potential energy, and the most extensive fields of coordination chemistry ${ }^{12,13}$ and organic light emitting diodes (OLED $)^{14}$ are the most widely studied, as shown in Figure 1.

The applications include the following: anionic specificity, self-assembly and binding; nano medicine ${ }^{15-20}$ and drug metabolism, ${ }^{21-24}$ and catalysts in biological systems. In addition, diaza-polycations are widely used

*e-mail: organicboron@ujs.edu.cn
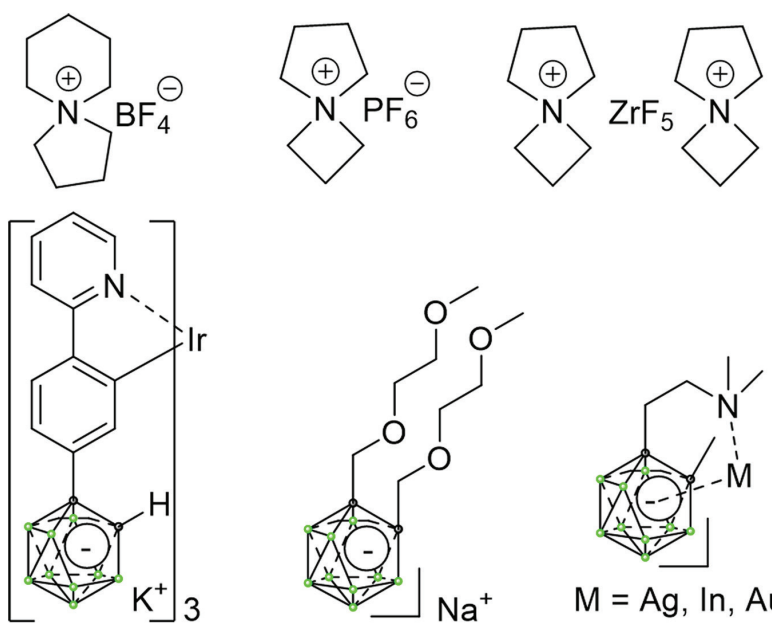

$\mathrm{M}=\mathrm{Ag}, \ln , \mathrm{Au}$

Figure 1. Azaspirodecanium salt and nido-carborane derivatives.

in agriculture applications. ${ }^{25-27}$ Polynitrogen compounds with ionic polynitrogen structure can play an effective role in promoting the growth of soil and plants. ${ }^{28-31}$

Nido-carborane has attracted little research attention, particularly in the fields of polycationic adducts and applications. In this study, a new azaspirodecanium was synthesized using a simple method based on the unique structure of nido-carborane combined with reaction characteristics to easily produce high yield carborane adducts. 


\section{Experimental}

All solvents and reagents were obtained commercially. Moisture sensitive reactions were performed under nitrogen atmosphere. Acetonitrile was distilled from calcium hydride before use. All glassware were torch flame or oven-dried and kept in a desiccator before use. Characterization of the products at each stage, by nuclear magnetic resonance (NMR) spectroscopy ${ }^{1} \mathrm{H}$ and ${ }^{13} \mathrm{C}$ spectra, were recorded on Bruker Avance spectrometer in $\mathrm{CD}_{3} \mathrm{OD}\left(400 \mathrm{MHz}\right.$ for ${ }^{1} \mathrm{H}, 100 \mathrm{MHz}$ for ${ }^{13} \mathrm{C}$ ); chemical shifts are expressed in ppm versus internal tetramethylsilane $(\mathrm{TMS})=0$ for ${ }^{1} \mathrm{H}$ and ${ }^{13} \mathrm{C}$. Coupling constants $(J)$ are given in Hz. Elemental analyses were performed using a Carlo Erba Instruments CHNS-O EA1108 analyzer. IR spectra of samples were recorded on an Nicolet avato-370 FT-IR analyzer using KBr disks. Elemental analysis (Carlo Erba Instruments CHNS-O EA1108 analyzer) and high resolution mass spectrometry (HRMS) (Jeol LTD JMS-HX 110/110A) were performed by the Uniplus company of the Republic of Korea.

General procedure for the synthesis of bis(4-azaspiro [3.4]octan-4-ium)-nido-ortho-caborane and bis(5-azaspiro [4.5]decan-5-ium)-nido-ortho-caborane

The general procedure for the nido-carborane potassium salt substitution reaction was as follows: nido-carborane potassium salt $(1.3 \mathrm{~g}, 0.6 \mathrm{mmol})$ was added to a solution of the 4-azaspiro[3.4] octan-4-ium chloride (1.9 g, $13 \mathrm{mmol}$ ) in distilled water $(15 \mathrm{~mL})$ and violent stirred at room temperature for few minutes. Then, mixture solution was filtered, washed with distilled water several times and dried to afford white solid.

\section{Bis(4-azaspiro[3.4]octan-4-ium)-nido-ortho-caborane}

A white solid (2.0 g, 93\%); IR (KBr) $v / \mathrm{cm}^{-1} 2513$ (B-H); ${ }^{1} \mathrm{H}$ NMR $\left(400 \mathrm{MHz}, \mathrm{CD}_{3} \mathrm{OD}\right) \delta 3.52-3.49$ (m, $4 \mathrm{H}), 3.41-3.38(\mathrm{~m}, 4 \mathrm{H}), 3.33-3.32(\mathrm{~d}, J 1.6 \mathrm{~Hz}, 2 \mathrm{H}), 3.20-$ 0.8 (br, 10H, (B-H)), 1.91-1.86 (m, 10H), 1.76-1.68 (m, $10 \mathrm{H}) ;{ }^{13} \mathrm{C}$ NMR (100 MHz, $\left.\mathrm{CD}_{3} \mathrm{OD}\right) \delta 62.40,60.41-60.36$ (t, $J$ 2.7, 21.14, 20.97, $20.91 \mathrm{~Hz}$ ); HRMS $m / z$, calcd. for $\mathrm{C}_{16} \mathrm{H}_{38} \mathrm{~B}_{9} \mathrm{~N}_{2}\left[\mathrm{M}^{+}\right]$: 355.2942, observed: 355.2935, calcd. for $\mathrm{C}_{16} \mathrm{H}_{38} \mathrm{~B}_{9} \mathrm{~N}_{2}$ : C 54.01, H 10.77, N 7.87, found: C 54.19, $\mathrm{H} 10.68$, N 7.81 .

\section{Bis(5-azaspiro[4.5]octan-5-ium)-nido-ortho-caborane}

A white solid (2.1 g, 91\%); IR (KBr) v / $\mathrm{cm}^{-1} 2537$ $(\mathrm{B}-\mathrm{H}) ;{ }^{1} \mathrm{H}$ NMR $\left(400 \mathrm{MHz}, \mathrm{CD}_{3} \mathrm{OD}\right) \delta 3.58-3.54(\mathrm{~m}$, $8 \mathrm{H}), 3.41-3.36(\mathrm{~m}, 8 \mathrm{H}), 3.33-3.32(\mathrm{~d}, J 1.6 \mathrm{~Hz}, 2 \mathrm{H}), 3.20-$ 0.8 (br, 10H, (B-H)), 2.22-2.18 (m, 8H), 1.91-1.86 (m,
$8 \mathrm{H}), 1.75-1.71(\mathrm{~m}, 4 \mathrm{H}) ;{ }^{13} \mathrm{C}$ NMR $\left(100 \mathrm{MHz}, \mathrm{CD}_{3} \mathrm{OD}\right)$ $\delta$ 60.91, 59.87-59.82 (t, $J 2.5 \mathrm{~Hz}), 25.79,19.31,19.25$, 18.62; HRMS $m / z$, calcd. for $\mathrm{C}_{20} \mathrm{H}_{46} \mathrm{~B}_{9} \mathrm{~N}_{2}\left[\mathrm{M}^{+}\right]$: 411.3571, observed: 411.3567, calcd. for $\mathrm{C}_{20} \mathrm{H}_{46} \mathrm{~B}_{9} \mathrm{~N}_{2}$ : C 58.32, H 11.26, N 6.80, found: C 58.38, H 11.31, N 6.56.

\section{Results and Discussion}

Azaspirodecanium is a tethered structure with a doublering model centered on a nitrogen atom. The structure is simple, stable, and has no conjugation effect. Therefore, it is widely used in the electrolyte of lithium batteries, ${ }^{32,33}$ as shown in Figure 1. First, 4-azaspiro[3.4]octan-4-ium chloride ${ }^{34,35}$ and 5-azaspiro[4.5]decan-5-ium chloride ${ }^{36}$ were obtained using conventional methods. According to the literature, ${ }^{37}$ potassium carbonate was used as an inorganic base to react with pyrrolidine under acetonitrile conditions, and 1,3-dichloropropane and 1,4-dichlorobutane were then added dropwise. The resulting compounds were cyclized using a one-pot method to obtain the ideal intermediate in 89 and $86 \%$ yield.

The facile synthesis of nido-carborane has been reported. ${ }^{38,39}$ Referring to these documents, we adopted a mild method which used ethanol as a solvent to give the nido-carborane potassium salt in ideal yield. Finally, the corresponding two intermediates were stirred in distilled water to form a target product bis(4-azaspiro[3.4]octan4-ium)-nido-ortho-caborane (93\%) and bis(5-azaspiro [4.5]decan-5-ium)-nido-ortho-caborane (91\%), as shown in Scheme 1.

Compounds bis(4-azaspiro[3.4]octan-4-ium)-nidoortho-caborane and bis(5-azaspiro[4.5]decan-5-ium)nido-ortho-caborane showed absorption bands in the infrared (IR) spectrum at 2540 and $2510 \mathrm{~cm}^{-1}$, as shown in Figure 2.

The diagnostic signals of the compounds were the azaspirodecanium peaks at $\delta 3.58$ and $1.68 \mathrm{ppm}$ in the ${ }^{1} \mathrm{H}$ NMR spectra, a broad signal caused by B-H peaks for the ortho-carborane units from $\delta 3.2-0.8 \mathrm{ppm}(\mathrm{br})$, and $\mathrm{C}-\mathrm{H}$ peaks at $\delta 3.33-3.25 \mathrm{ppm}$. The ${ }^{13} \mathrm{C}$ NMR spectra showed peaks at $\delta 62.40-60.91 \mathrm{ppm}$ for the ortho-carborane $\mathrm{C}-\mathrm{H}$ bond.

And then, ${ }^{11} \mathrm{~B}$ NMR spectroscopy was performed. Usually, the characteristic fluorine peak of carborane appears as a double or multi-peak at 20-(-20) ppm. When nido-ortho-carborane is complexed with bis(4-azaspiro [3.4]octan-4-ium) or bis(5-azaspiro[4.5]decan5 -ium), the peak changes to a broad double peak, from bis(4-azaspiro[3.4] octan-4-ium)-nido-ortho-caborane (-10.94 ppm) and bis(5-azaspiro[4.5]decan-5-ium)-nidoortho-caborane (-10.96 ppm), as shown in Figure 3. Under 


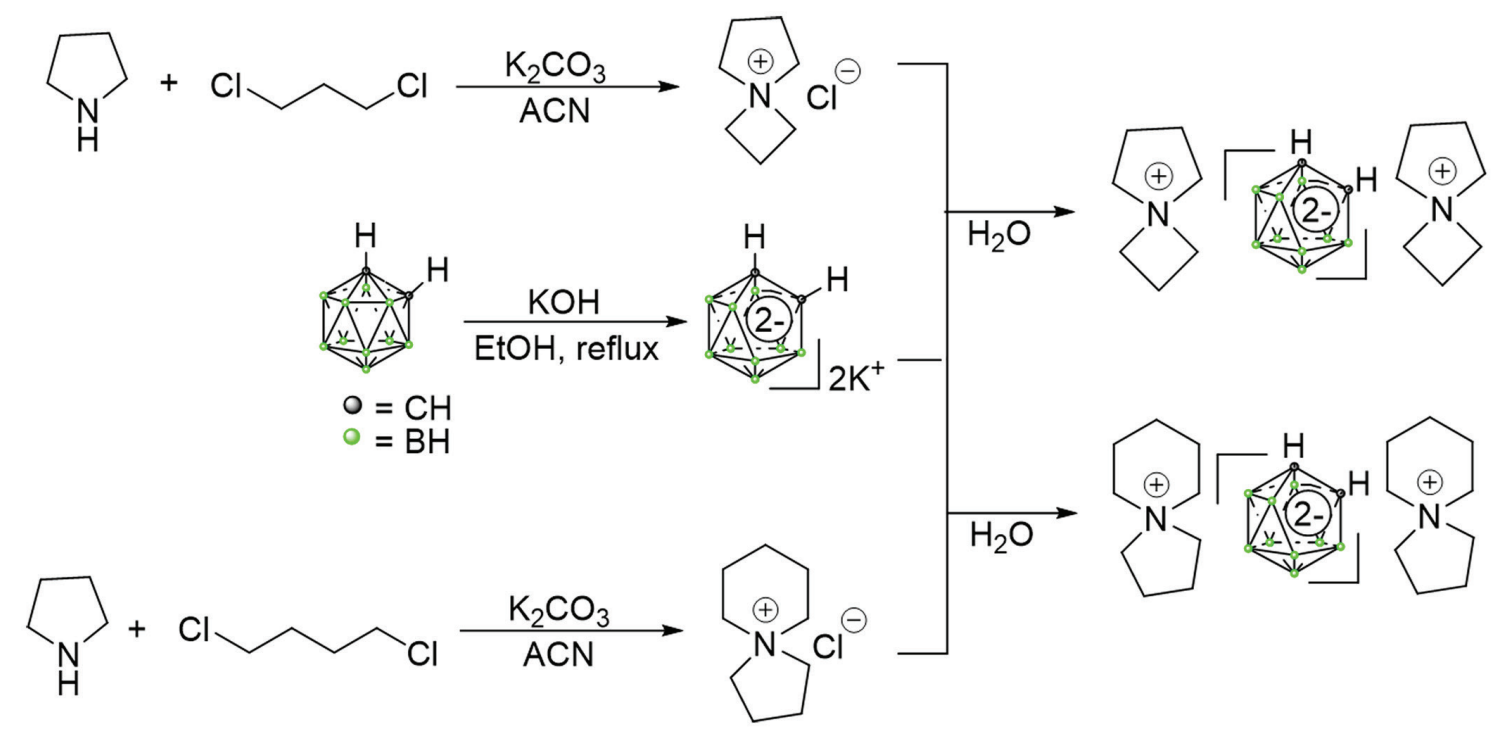

Scheme 1. Synthesis of bis(4-azaspiro[3.4]octan-4-ium)-nido-ortho-caborane and bis(5-azaspiro[4.5]decan-5-ium)-nido-ortho-caborane.

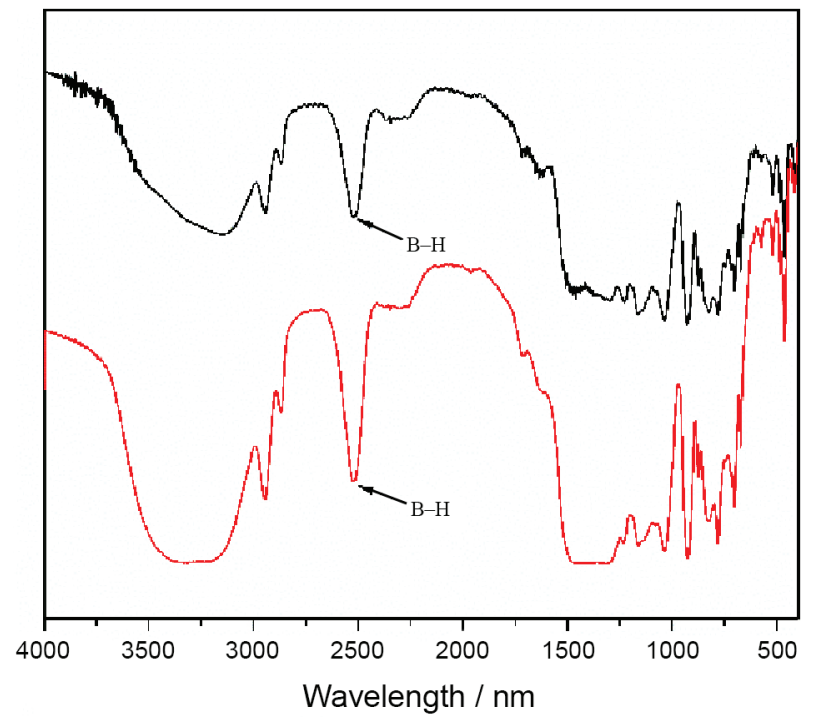

Figure 2. IR spectrum of azaspirodecanium-nido-ortho-caboranes.

the influence of nido-ortho-carborane, bis(4-azaspiro [3.4]octan-4-ium)-nido-ortho-caborane and bis(5-azaspiro[4.5]decan-5-ium)-nido-ortho-caborane did not show obvious changes in ${ }^{11} \mathrm{~B}$ NMR, but there were some subtle fluctuations in the NMR shifts (see Supplementary Information section).

\section{Conclusions}

A new shape of nido-carborane-substituted bipolarfunction derivatives, such as bis(4-azaspiro[3.4]octan4-ium)-nido-ortho-caborane and bis(5-azaspiro [4.5]decan-5-ium)-nido-ortho-caborane, were synthesized. The coupling of the azaspirodecanium salt with nido-carborane potassium salt to produce the target compounds proceeded successfully, which were further one-top substituted to produce the final compound in
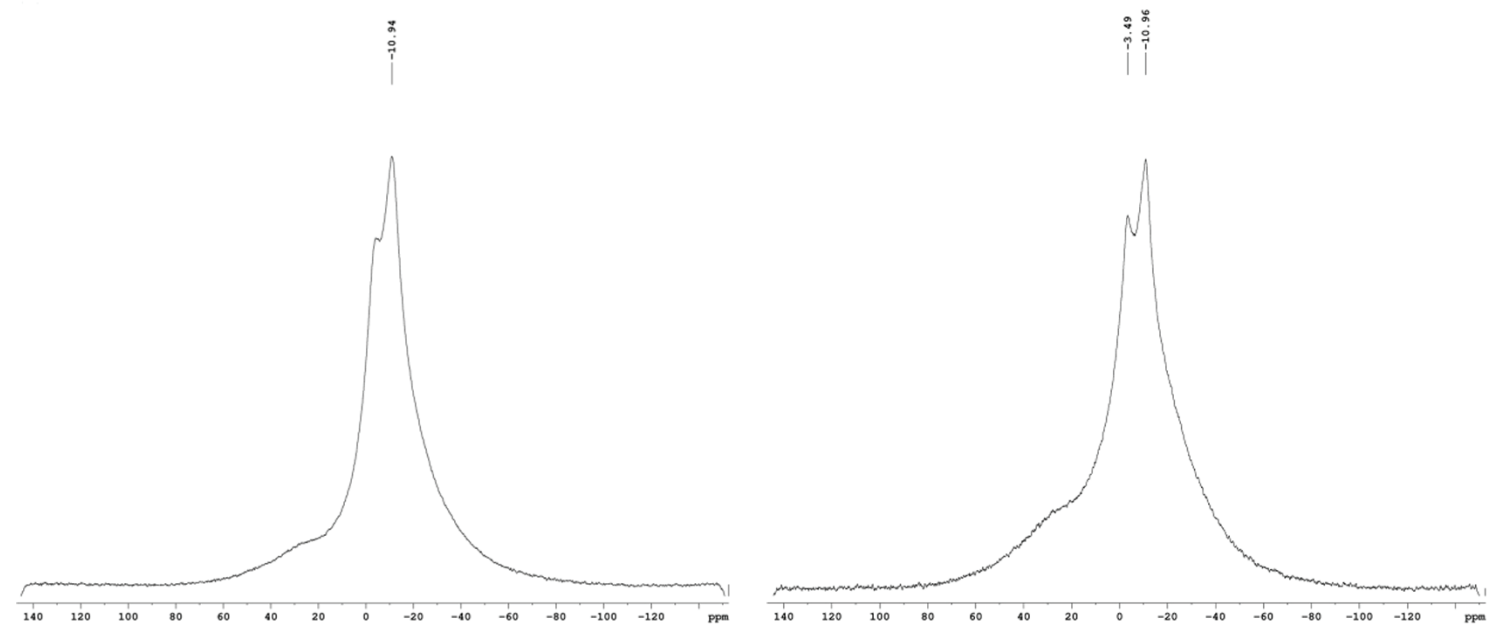

Figure 3. ${ }^{11} \mathrm{~B}$ spectra of azaspirodecanium-nido-ortho-caboranes. 
good yield. The effects of the synthesized compounds on the chemical reaction showed outstanding stability. In particular, both compounds could be generated under relatively gentle conditions, e.g., in water. Further studies to characterize the physical properties of bis(azaspirodecanium)-nido-carborane are currently underway.

\section{Supplementary Information}

Supplementary information (Figures S1-S10) is available free of charge at http://jbcs.sbq.org.br as PDF file.

\section{Acknowledgments}

This study was supported financially by the talent introduction of scientific research foundation of Jiangsu University (grant No. 5501290005).

\section{References}

1. Erokhina, S. A.; Stogniy, M. Y.; Suponitsky, K. Y.; Kosenko, I. D.; Sivaev, I. B.; Bregadze, V. I.; Polyhedron 2018, 153, 145.

2. Hosmane, N. S.; Grimes, R. N.; Inorg. Chem. 1979, 18, 2286.

3. Teixidor, F.; Vinas, C.; Sillanpaa, R.; Kivekas, R.; Casabo, J.; Inorg. Chem. 1994, 33, 2645.

4. Tanaka, T.; Araki, R.; Saido, T.; Abe, R.; Aoki, S.; Eur. J. Inorg. Chem. 2016, 20, 3330.

5. Kurihara, R.; Nohtomi, A.; Wakabayashi, G.; Sakurai, Y.; Tanaka, H.; J. Nucl. Sci. Technol. 2019, 56, 70.

6. Hsiao, M.-C.; Jiang, S.-H.; Appl. Radiat. Isot. 2019, 143, 79.

7. Wang, F.; Chen, L.; Zhang, D.; Jiang, S.; Shi, K.; Huang, Y.; Li, R.; Xu, Q.; J. Drug Targeting 2014, 22, 849.

8. Rodriguez, C.; Carpano, M.; Curotto, P.; Thorp, S.; Casal, M.; Juvenal, G.; Pisarev, M.; Dagrosa, M. A.; Radiat. Environ. Biophys. 2018, 57, 143.

9. Zhao, T.; Mao, G.; Mao, R.; Zou, Y.; Zheng, D.; Feng, W.; Ren, Y.; Wang, W.; Zheng, W.; Song, J.; Chen, Y.; Yang, L.; Wu, X.; Food Chem. Toxicol. 2013, 55, 609.

10. Yi, C. X.; Zhong, H.; Tong, S. S.; Cao, X.; Firempong, C. K.; Liu, H. F.; Fu, M.; Yang, Y.; Feng, Y. S.; Zhang, H. Y.; Int. J. Nanomed. 2012, 7, 5067.

11. Huo, M. R.; Zhao, Y.; Satterlee, A. B.; Wang, Y. H.; Xu, Y.; Huang, L.; J. Controlled Release 2016, 245, 81.

12. Thomas, W.; James, T. S.; Mohammad, R. P.; Russell, N. G.; Inorg. Chem. 1987, 26, 3116.

13. Xu, T.-T.; Cao, K.; Zhang, C.-Y.; Wu, J.; Jiang, L.; Yang, J.; Chem. Commun. 2018, 54, 13603.

14. Shi, C.; Sun, H.; Tang, X.; Lv, W.; Yan, H.; Zhao, Q.; Wang, J.; Huang, W.; Angew. Chem., Int. Ed. 2013, 52, 13434.
15. Cao, X.; Deng, W.; Wei, Y.; Su, W.; Yang, Y.; Wei, Y.; Yu, J.; Xu, X.; Int. J. Nanomed. 2011, 6, 3335.

16. Fei, X.; Zhu, Z.-Y.; Chen, X.; Hua, X.-Q.; Shan, X.-H.; Zhang, Y.; Gu, N.; Pharm. Res. 2012, 29, 1087.

17. Chen, Y.; Yuan, L.; Zhou, L.; Zhang, Z. H.; Cao, W.; Wu, Q.; Int. J. Nanomed. 2012, 7, 4581.

18. Cao, X.; Deng, W.; Fu, M.; Wang, L.; Tong, S. S.; Wei, Y. W.; Xu, Y.; Su, W. Y.; Xu, X. M.; Yu, J. N.; Int. J. Nanomed. 2012, 7, 753 .

19. Shen, S.; Wu, L.; Xie, M.; Shen, H.; Qi, X.; Yan, Y.; Ge, Y.; Jin, Y.; Int. J. Pharm. 2015, 486, 380.

20. Zhu, Y.; Wang, M.; Zhang, J.; Peng, W.; Firempong, C.K.; Deng, W.; Wang, Q.; Wang, S.; Shi, F.; Yu, J.; Arch. Pharmacal. Res. 2015, 38, 512.

21. Sheng, J.; Tian, X.; Xu, G.; Wu, Z.; Chen, C.; Wang, L.; Pan, L.; Huang, C.; Pan, G.; Drug Metab. Dispos. 2015, 43, 63.

22. Feng, W.; Zhao, T.; Mao, G.; Wang, W.; Feng, Y.; Li, F.; Zheng, D.; Wu, H.; Jin, D.; Yang, L.; Wu, X.; PLoS One 2015, 10, e0125952.

23. Qian, Q.; Li, S. L.; Sun, E.; Zhang, K. R.; Tan, X. B.; Wei, Y. J.; Fan, H. W.; Cui, L.; Jia, X. B.; J. Pharm. Biomed. Anal. 2012, 66, 392.

24. Sun, E.; Xu, F. J.; Qian, Q.; Li, C.; Tan, X. B.; Jia, X. B.; Nat. Prod. Res. 2014, 28, 1525.

25. Yan, J. K.; Wang, Y. Y.; Qiu, W. Y.; Ma, H. L.; Wang, Z. B.; Wu, J. Y.; Crit. Rev. Food Sci. Nutr. 2018, 58, 2416.

26. Feng, Y. S.; Zhu, Y.; Wan, J. Y.; Yang, X.; Firempong, C. K.; Yu, J. N.; Xu, X. M.; J. Funct. Foods 2018, 44, 137.

27. Rashid, M. T.; Hashim, M. M.; Wali, A.; Ma, H. L.; Guo, L. N.; Jian, X.; J. Food Saf. Food Qual. 2018, 69, 19.

28. Zhang, D.; Du, M. Z.; Wei, Y.; Wang, C. T.; Shen, L. Q.; J. Food Biochem. 2018, 42, 5.

29. Xiong, F.; Dai, C. H.; Hou, F. R.; Zhu, P. P.; He, R. H.; Ma, H. L.; Czech J. Food Sci. 2018, 36, 88.

30. Ayim, I.; Ma, H.; Alenyorege, E. A.; Ali, Z.; Donkor, P. O.; Zhou, C.; J. Food Meas. Charact. 2018, 12, 2695.

31. Zuo, Z. Y.; Li, X. G.; Xu, C.; Yang, J. J.; Zhu, X. C.; Liu, S. Q.; Song, F. B.; Liu, F. L.; Mao, H. P.; Plant, Soil Environ. 2017, 63,348 .

32. Wang, G.; Fu, X. L.; Wang, J. J.; Guan, R.; Tang, X. J.; Curr. Cancer Drug Targets 2017, 17, 17.

33. Baseren, S. C.; Erdogmus, A.; Gul, A.; J. Organomet. Chem. 2018, 866, 105.

34. Jonsson, E.; Armand, M.; Johansson, P.; Phys. Chem. Chem. Phys. 2012, 14, 6021.

35. Jin, G. F.; Jin, F.; Wang, K.; Zheng, B.; Fu, Y.; Jin, Z.; Liu, J.; CN pat. 106117217, 2016.

36. Arnott, E. A.; Crosby, J.; Evans, M. C.; Ford, J. G.; Jones, M. F.; Leslie, K. W.; McFarlane, I. M.; Sependa, G. J.; WO pat. 2008053221 A2, 2008. 
37. Kitamura, M.; Yamamura, S.; Kobayashi, H.; Yamamoto, M.; Tada, K.; Hioki, K.; Yamada, K.; Kunishima, M.; Chem. Lett. 2014, 43, 1593.

38. Vorberg, R.; Carreira, E. M.; Muller, K.; ChemMedChem. 2017, 12,431 .
39. Timofeev, S. V.; Zhidkova, O. B.; Prikaznova, E. A.; Sivaev, I. B.; Semioshkin, A.; Godovikov, I. A.; Starikova, Z. A.; Bregadze, V. I.; J. Organomet. Chem. 2014, 757, 21.

Submitted: February 15, 2019

Published online: July 3, 2019 